\title{
Challenges and Open Questions in Soft Consensus Models
}

\author{
F.J. Cabrerizo \\ Dept. of Computer Systems and \\ Software Engineering \\ UNED \\ Madrid, 28040, Spain \\ cabrerizo@issi.uned.es
}

\author{
F. Chiclana \\ Centre for Computational Intelligence \\ School of Computing \\ De Montfort University \\ Leicester, LE1 9BH, UK \\ chiclana@dmu.ac.uk
}

\author{
M.R. Ureña, E. Herrera-Viedma \\ Dept. of Computer Sciences and \\ Artificial Intelligence \\ University of Granada \\ Granada, 18071, Spain \\ raquel@decsai.ugr.es \\ viedma@decsai.ugr.es
}

\begin{abstract}
In group decision making problems, given the importance of obtaining an accepted solution by the whole group, the consensus has attained a great attention and it is virtually a major goal of these problems. Consensus, as traditionally meant to be a full and unanimous agreement, is often not reachable in practice. A more realistic approach is to use softer consensus measures, which assess the consensus degree in a more flexible way, and therefore reflect the large spectrum of possible partial agreements, guiding the consensus process until widespread agreement is achieved among experts. In particular, the interpretation of the consensus based on the concept of fuzzy majority has been used in the most of the consensus models proposed in the literature, as it is more human-consistent and suitable for reflecting human perceptions of the meaning of consensus. However, there are still some open questions to be addressed. In this paper, we are going to highlight some issues in order to focus researcher's attention on new problems that arise when using consensus models based on soft consensus measures in real-world applications.
\end{abstract}

\section{INTRODUCTION}

A group decision making (GDM) problem concerns a situation in which there is a group of individuals (experts) who present their testimonies which basically concern their opinions on some alternatives, topics, courses of action, etc., in question [1], [2]. In this situation, we can find collaboration, competitiveness, compatible approaches or incompatible proposals involving many different environments (companies, politics, teaching, etc.). The aim is to reconcile differences of opinions expressed by individual experts to find a solution, which is best acceptable by the group of experts as a whole. As consensus decisions are indeed better valued by the people, it is preferable that the group of experts achieves a great agreement among their opinions before obtaining a solution. Due to this fact, consensus has become a major area of research in GDM.

Consensus has been a much talked about word for centuries, maybe millennia since people have always been aware of its importance for arriving at proper decisions which have had a long lasting impact on life of groups of people, countries or even civilizations. Needles to say that the growing complexity of the present world has made the word to be used so frequently nowadays [3].
A crucial point here is the very meaning of consensus. Consensus can be meant in various ways and in various contexts in GDM. First, it refers to the state of agreement in a group in the sense that the individuals exhibit a state of common feeling as to the values in question. Strictly speaking, consensus has been meant from this perspective as a full and unanimous agreement [4], [5], though it has been deemed questionable if such a state is possible in virtually all real world situations [6]. The second sense of this word, which is related to the first sense given above, is meant as a way to reach consensus. This involves an evolution of the testimonies of the group towards consensus with respect to their testimonies; this evolution can be free or facilitated (moderated) by a special individual [1], [7]. It is easy to see that the second sense of consensus does involve the first sense because the process of reaching an agreement must be related to some assessment of what, and to which extent the current agreement within the group exists.

As we have already mentioned, consensus has been traditionally meant as a full and unanimous agreement. For example, several authors have introduced consensus measures assuming values in-between 0 (no consensus or partial consensus) and 1 (full consensus) [4], [5]. However, the human perception of consensus is typically "softer", and people are generally willing to accept that consensus has been reached when most actors agree on the preferences associated to the most relevant alternatives. A milestone was here a special issue of the influential Synthese journal (cf. Loewer [8]). Among many papers therein, the most relevant for our purpose is that by Loewer and Laddaga [9], who have clearly made the case for a soft concept of consensus saying that:

... It can correctly be said that there is a consensus among biologists that Darwinian natural selection is an important cause of evolution though there is currently no consensus concerning Gould's hypothesis of speciation. This means that there is a widespread agreement among biologists concerning the first matter but disagreement concerning the second ...

A crisp majority as, e.g., more than $75 \%$ would not evi- 
dently reflect the very essence of the above given quotation. This statement suggests that a fuzzy majority is appropriate, and that it makes sense to speak about a degree of consensus, or a distance from (ideal) consensus.

According to Loewer and Laddaga, Kacprzyk and Fedrizzi introduced the concept of a fuzzy majority using Zadeh's fuzzy linguistic quantifier to compute soft consensus measures in [6], [10], [11].

Then, the classical operational definition of consensus is expressed by a linguistically quantified proposition as:

"Most $(Q 1)$ of the important $(B)$ individuals agree as to almost all $(Q 2)$ relevant $(I)$ alternatives"

where: $Q 1$ and $Q 2$ are fuzzy linguistic quantifiers [12], e.g., "most" and "almost all", and $B$ and $I$ stand for fuzzy sets denoting the importance/relevance of the individuals and alternatives. Therefore, the notion of soft consensus introduced by Kacprzyk and Fedrizzi is based on fuzzy sets theory [13].

These Kacprzyk and Fedrizzi's works constitute the basis of many soft consensus models proposed later, which assess the consensus degree in a more flexible way and, therefore, reflect the large spectrum of possible partial agreements and guide the consensus process until widespread agreement (not always full) is achieved among experts. However, despite the great efforts done to develop new soft consensus models in GDM problems, there still exist some prospects and open questions which have to be faced.

The aim of this paper is to analyze new problems that arise when using soft consensus models in real-world applications in order to focus the researcher's attention on them.

The rest of the paper is structured as follows. In Section II, we make a brief review of different basic concepts related to soft consensus models. Section III analyzes the challenges an open questions that should be faced in order to improve the results of the soft consensus models in GDM problems. Finally, in Section IV, we draw some conclusions.

\section{Preliminaries}

In this section, we describe both the classical GDM problems and the usual consensus process.

\section{A. GDM Problem}

A classical GDM problem may be defined as a decision situation where [14], [15]:

- There exist a group of two or more experts, $E=$ $\left\{e_{1}, e_{2}, \ldots, e_{m}\right\}(m \geq 2)$, characterized by their own ideas, attitudes, motivations and knowledge.

- There is a problem to solve in which a solution must be chosen among a set of possible alternatives, $X=$ $\left\{x_{1}, x_{2}, \ldots, x_{n}\right\}(n \geq 2)$.

- The experts try to achieve a common solution.

In a fuzzy context, the objective is to classify the alternatives from best to worst, associating with them some degrees of preference expressed in the $[0,1]$ interval.

The solution for a GDM problem is derived either from the individual preferences provided by the experts, without constructing a social opinion, or by computing first a social opinion and then using it to find a solution [16]. These two approaches are respectively called direct and indirect approaches, and within both approaches, the process for reaching a solution of the GDM problem is composed by two steps [15], [17]:

1) Aggregation phase. In this phase, all preferences given by the experts must be aggregated into only one preference structure. This aggregation is carried out by means of particular aggregation operators that are usually defined for this purpose.

2) Exploitation phase. This phase uses the information produced in the aggregation phase to identify the solution set of alternatives for the problem.

The above selection process does not necessarily check any agreement among the experts before obtaining the solution for the GDM problem. Therefore, this process can lead sometimes solutions that are not well accepted by some experts in the group [1], [2], because they could consider that their preferences have not been taken into account properly to obtain the solution, and hence, they might reject it. To avoid this situation, it is advisable that experts carry out a consensus process, where the experts discuss and modify their preferences gradually to achieve a sufficient agreement before applying the selection process. For this reason, GDM problems are usually faced by applying a consensus process and a selection process before a final solution can be given [18], [19].

In the following subsection, we will review the structure of a typical consensus process and analyze their phases.

\section{B. Consensus Process}

There are two approaches in the formulation of consensus process. The first, traditional, is the one started by French, Harary, etc., [20], [21], [22] in which the process is modeled by using matrix calculus or Markov chains to model the time evolution of changes of opinions toward consensus. The second, more promising in practice, is the one in which there is a moderator. In the following, we describe the second one as it is the most used in the literature.

A consensus process is defined as a dynamic and iterative group discussion process, coordinated by a moderator helping experts bring their opinions closer. At the beginning of every GDM problem, the set of experts has diverging opinions, then the consensus process is applied and, in each step, the degree of existing consensus among experts' opinions is measured. If the consensus degree is lower than a specified threshold, the moderator would urge experts to discuss their opinions further in an effort to bring them closer. Otherwise, the moderator would apply the selection process in order to obtain the final consensus solution to the GDM problem. The moderator knows the agreement in each moment of the consensus process by means of the computation of some consensus measures.

The consensus process can be divided in several steps which are graphically depicted in Fig. 1:

1) Firstly, the problem to be solved is presented to the 


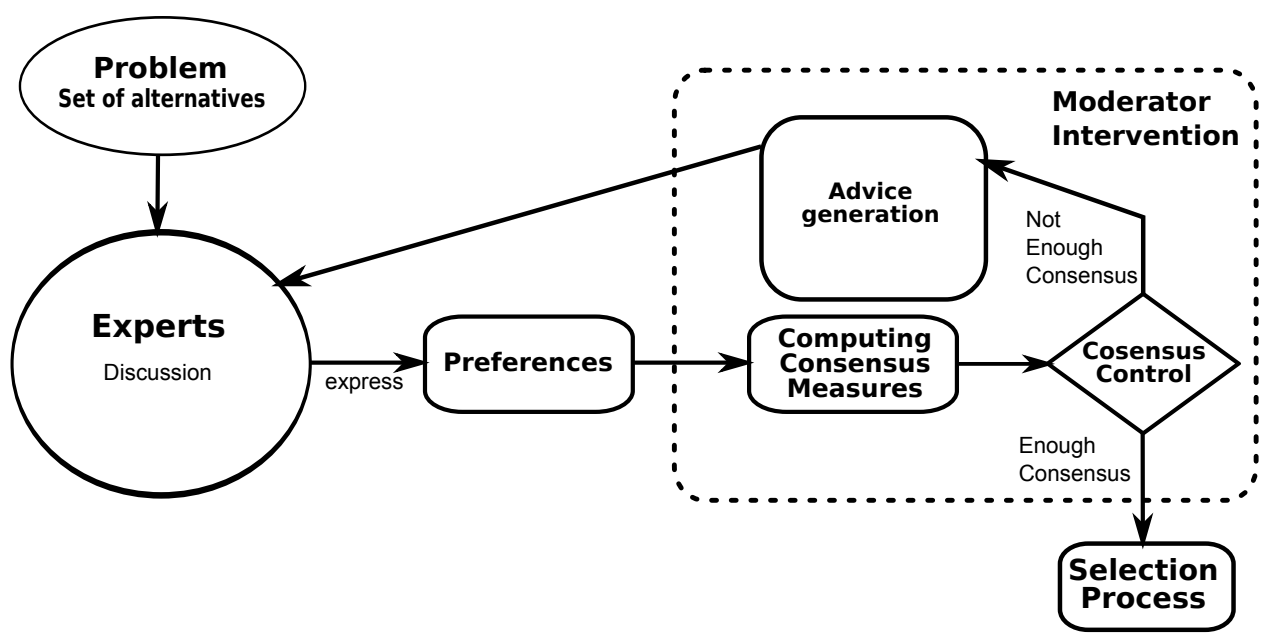

Fig. 1. Consensus Process Scheme

experts, along with the different alternatives among they have to choose the best one.

2) Then, experts can discuss and share their knowledge about the problem and alternatives in order to facilitate the process of latterly expressing their opinions.

3) Experts provide their preferences about the alternatives in a particular preference representation format.

4) The moderator receives all the experts' preferences and computes some consensus measures that will allow him to identify if an enough consensus state has been reached or not.

5) If an enough consensus state has been reached, the consensus process stops and the selection process begins. Otherwise, we can apply an advice generation step where the moderator, with all the information that he/she has (all preferences expressed by experts, consensus measures and so on) can prepare some guidance and advice for experts to more easily reach consensus. Note that this step is optional and is not present in every consensus model.

6) Finally, the advice is given to the experts and the first round of consensus is finished. Again, experts must discuss their opinions and preferences in order to approach their points of view (Step 2).

Once we have described the typical consensus process, we are ready to point out the challenges that must be faced to improve the performance of the consensus process within GDM problems.

\section{Prospects And Open Questions}

In this section, some issues will be highlighted in order to focus researcher's attention on new problems that arise when using consensus models based on soft consensus measures in real-world applications. There are still some challenges and open questions to be addressed.

\section{A. Advice Generation}

Some consensus models incorporate a feedback mechanism substituting the moderator's actions, avoiding the possible subjectivity that he/she can introduce, and giving advice to the experts to find out the changes they need to make in their opinions to obtain a solution with certain consensus degree [23], [24]. Most of these automatic consensus models existing in the literature present the same behavior in all discussion rounds of the consensus reaching process. However, when the level of agreement between the experts is "high", a few number of changes of opinions from some of the experts might lead to consensus in a few discussion rounds. On the contrary, when the level of agreement among the experts is "low", a high number of changes of opinions and many group discussion rounds might be necessary for consensus to be achieved. In other words, the number of changes in different stages of a consensus process is clearly related to the actual level of agreement. Following this idea, an adaptive consensus model has been proposed by Mata et al. in [25]. It is based on a refinement process of the consensus process that allows to increase the agreement and reduce the number of experts' preferences that should be changed after each consensus round, adapting the search for the furthest experts' preferences to the existent agreement in each round of consensus. To do so, three levels of agreement are distinguished: very low, low and medium consensus. Each level of consensus involves to carry out the search for the furthest preferences in a different way: when the consensus degree is very low, it will search for the furthest preferences on all experts, while if the consensus degree is medium, the search will be limited to the furthest experts. This adaptive model increases the convergence toward the consensus and reduces the number of rounds to reach it.

However, there are still some open questions about adaptive consensus models: (i) it would be desirable to extend adaptive consensus models to decision making contexts managing different formats of preference representation, and (ii) to study a mechanism to guarantee the convergence of the adaptive 
consensus models is still a challenge.

\section{B. Trust Based Consensus Models}

Situations in which the group of experts vary over time are quite common in real decision processes: a new expert could incorporate to the process, some experts could leave it or a large group of experts could be simplified in order to minimize communications and ease the computation of solutions. We can think of a large group of people that share a common interest and that form an on-line community about their topic of interest. In those situations, to carry out a proper consensus process is a difficult task. For example, not every member of the community is willing to participate and contribute to solve the problem. Usual approaches involve using opinion polls and forums, but those methods do not offer methods to control the consensus of the process and the discussions (which tends to be very disperse due to the large number of speakers). To address these situations, a new consensus model has been proposed in [26]. This approach follows an scheme based on the one presented in Section II-B, but it incorporates several important differences in order to deal with a large number of experts. The most important difference is the inclusion of a previous step, prior to the consensus phase, in which the large group of experts is simplified into a "selected experts group" or spokespersons by using a clustering algorithm, trying to maintain the diversity on the opinions of the whole group. Once this simplification is made, the experts that have not been selected will provide information about the trust that the selected experts inspire to them, thus creating a trust network [27]. After this initial step the consensus process begins, but only the selected experts that are allowed to take part in the process. Thus it is possible to carry out a proper consensus process, with discussion among the smaller group of spokespersons. At each consensus round the non-selected experts will be able to change their trust evaluations if their opinions about the selected experts have changed. Once a proper level of consensus is reached, the selection process begins, the opinions of the selected experts are aggregated, and the final solution is obtained.

The use of trust degrees in consensus processes is still in an early stage of development and several future challenges have still to be solved: (i) development of automatic process to compute the trust degrees in decision making, (ii) to introduce in the consensus models advanced trust management models that have been used satisfactorily in other frameworks, as recommender systems [28], and (iii) using the trust criteria as other possibility to guide the consensus process.

\section{Visualization and Verbalization}

The application of the latest technologies has extended the opportunities in decision making and has allowed to carry out consensus processes in situations that we cannot correctly address previously. For example, nowadays, it possible to carry out consensus processes among several experts that are located in different countries around the world using mobile or web technologies. However, it is important to remark that even with the adoption of new communication technologies, there is still an important need of new collaboration and information tools for the experts being able to solve decision making problems where they cannot meet together with the other experts [29]. A particular problem that arises in many consensus processes for GDM when experts do not have the possibility of gathering together is that experts may not have a clear idea about the current consensus status among all the experts involved in the decision process. In usual decision making models, where experts gather together to discuss their opinions about the different alternatives, it is relatively easy to determine which experts have similar opinions just by attending to the discussions among experts, and thus, experts may join or form different groups to better discuss and reason out about the pros and cons of every alternative. Additionally, when experts are able to determine the consensus state of the decision making process it is more easy for them to influence the other experts and to detect if some experts are trying to bias the consensus process. However, in the cases where direct communication is not possible, experts will probably need some assistance to establish connections among them and to obtain a clear view of the consensus process progress.

To solve these problems, both (i) visualization and (ii) verbalization can be used. One the one hand, new techniques and tools to automatically generate high level information and simple consensus diagrams about the consensus state in the decision problem need to be developed. As visual elements do have a great potential to influence experts in decision processes, these consensus diagrams, when presented to the experts, will allow them to have a more profound and clear view about the current consensus process and about which experts have similar or different opinions about the alternatives. Additionally, the use of these diagrams can help the experts to detect if other experts are trying to bias the consensus process. On the other hand, though visualization is a powerful means of communication in virtually all human centric systems, other means of communications may be employed, notably verbalization that can take full advantage of the use of natural language which is anyway extensively used in the area and it is well documented [30].

\section{Experts' Importance}

In GDM, there are situations where the information handled by the experts is not equally important [31], [32]. For example, when a group of medical experts expresses its opinions on the possible illness that a patient presents, its diagnostics must not be considered with equal relevance, given that, there will be medical experts with more experience or with more study years than others, and, therefore, all the opinions shall not be equally reliable. But, a final and global diagnostic must be made using the initial and individual diagnostics. To model such situations, the most usual approach in the literature consists in the assignation of weight values to the experts, which reflect the relevance of the expert in the group. Consensus models have taking into account the heterogeneity of the experts when aggregating the experts' opinions to obtain 
the collective preference [33], [34], [35], but not when advising to the experts how to change their preferences to increase the consensus level. Then, we think that it is important to develop consensus models taking into account the experts' importance weights not only to aggregate the experts' preferences but also when advising experts to change their preferences. Thus, new feedback mechanisms that adjust the amount of advice required by each expert depending on his/her own knowledge level about the problem should be developed. It seems reasonable that experts with lower importance or knowledge level will need more advice than those experts that previously have at their disposal a large amount of information to make good decisions. Therefore, the consensus model should generate the recommendations in a different way depending on the expert's knowledge level in order to increase the agreement in the next consensus round.

\section{E. Dynamic Decision Contexts}

Because good decisions depend on the conditions of the future, and because conditions vary over time, to make a good decision requires judgments of what is more likely or more preferred over different time periods. According to Saaty [36], there are at least three ways to deal with dynamic decisions. One is to include in the structure itself different factors that indicate change in time such as scenarios and different time periods and then carry out paired comparisons with respect to the time periods. The second is to do paired comparisons as rates of relative change with respect to time. This is done at different points of time as representatives of intervals to which they belong. These intervals can have different lengths. For each representative point one needs to provide a pairwise judgment about the relative rate of change of one alternative over another and derive mathematical functions for that matrix of comparisons for one of the time periods. The third is to use functions for the paired comparisons and derive functions from them.

Time dependent decision-making is a subject that we need today. At a minimum they are needed in technical design problems in which the influences of several tangible design factors change over time and tradeoffs must be made among them to enable the system to respond differently and continuously over the time of its operation. In such a way, new consensus models in time-dependent GDM should be developed.

\section{F. Persuasion}

Once of the moderator's task in the consensus process is to produce some advice for those experts that should change their opinions in order to increase the level of consensus. However, when experts' opinions are different among them, it necessary that some of the experts change their mind in order to achieve the consensus, and the moderator is who has to persuade them to do it. However, persuading people is a difficult task. According to professor Cialdini [37], although there are thousands of different tactics that people employ to influence others, the majority fall within six basic categories:
1) Social proof. People will do thing that they see other people are doing.

2) Authority. People will tend to obey authority figures, even if they are asked to perform objectionable acts.

3) Linking. People are easily persuaded by other people that they like.

4) Scarcity. Infrequent items or resources will generate demand.

5) Consistency. If people commit, verbally or in writing, to and idea or goal, they are more likely to honor that commitment.

6) Reciprocation. People tend to return a favor.

Each of these categories is governed by a fundamental psychological principle that directs human behavior and gives the tactics power of persuasion [37]. These principles of persuasion or weapons of influence can be used as a support for the consensus process as they address the use communication in order to change attitudes, beliefs or the behavior of others in a voluntary manner avoiding the use of coercion. Thus, we propose the introduction of some psychology concepts (or principles or persuasion) in the consensus process.

\section{CONCLUSION}

The application of consensus models based on soft consensus measures in GDM problems has received great attention from the research community for the last years. Since Kacprzyk proposed the concept of soft consensus measures, many papers about this research topic have been published. However, there still exist some prospects and open questions which have to be faced when dealing with GDM problems. In this paper, we have highlighted some issues and challenges which have to be addressed by the researchers in order to improve the performance of the consensus process within GDM problems.

\section{ACKNOWLEDGMENT}

This work has been developed with the financing of FEDER funds in FUZZYLING-II Project TIN2010-17876, the Andalusian Excellence Projects TIC-05299 and TIC-5991, and "Programa José Castillejo 2011 (JC2011-0002)".

\section{REFERENCES}

[1] C. T. Butler and A. Rothstein, On Conflict and Consensus: A Handbook on Formal Consensus Decision Making, Takoma Park, 2006.

[2] S. Saint and J. R. Lawson, Rules for Reaching Consensus: A Modern Approach to Decision Making, Jossey-Bass, 1994.

[3] E. Herrera-Viedma, J. L. Garcia-Lapresta, J. Kacprzyk, M. Fedrizzi, H. Nurmi and S. Zadrozny, Consensual Processes, Studies in Fuzziness and Soft Computing, Volume 267/2011, Springer, 2011.

[4] J. C. Bezdek, B. Spillman and R. Spillman, "A fuzzy relation space for group decision theory", Fuzzy Sets and Systems, vol. 1, pp. 255-268, 1978.

[5] B. Spillman, R. Spillman and J. C. Bezdek, "A fuzzy analysis of consensus in small groups', in Fuzzy Automata and Decision Processes, P. P. Wang and S. K. Chang, Eds. Amsterdam: North-Holland, 1980, pp. $331-356$.

[6] J. Kacprzyk and M. Fedrizzi, "'Soft' consensus measures for monitoring real consensus reaching processes under fuzzy preference", Control and Cybernetics, vol. 15, pp. 309-323, 1986. 
[7] P. Eklund, A. Rusinowska and H. D. Swart, "Consensus reaching in committees", European Journal of Operational Research, vol. 178, pp. 185-193, 2007.

[8] B. Loewer, "Special issue on consensus", Synthese, vol. 62, pp. 1-122, 1985.

[9] B. Loewer and R. Laddaga, "Destroying the consensus", Synthese, vol. 62, pp. 79-96, 1985.

[10] J. Kacprzyk and M. Fedrizzi, "A 'soft' measure of consensus in the setting of partial (fuzzy) preferences", European Journal of Operational Research, vol. 34, pp. 316-325, 1988.

[11] J. Kacprzyk and M. Fedrizzi, "A 'human-consistent' degree of consensus based on fuzzy logic with linguistic quantifiers", Mathematical Social Sciences, vol. 18, pp. 275-290, 1989.

[12] L. A. Zadeh, "A computational approach to fuzzy quantifiers in natural languages", Computers \& Mathematics with Applications, vol. 9, 149184, 1989.

[13] L. A. Zadeh, "Fuzzy sets", Information and Control, vol. 8, 338-353, 1965.

[14] S. J. Chen and C. L. Hwang, Fuzzy Multiple Attribute Decision-Making: Methods and Applications, New York: Springer-Verlag, 1992.

[15] J. C. Fodor and M. Roubens, Fuzzy Preference Modelling and Multicriteria Decision Support, Kluwer Academic Publishers, 1994.

[16] J. Kacprzyk, "Group decision making with a fuzzy linguistic majority", Fuzzy Sets and Systems, vol. 18, pp. 105-118, 1986.

[17] M. Roubens, "Fuzzy sets and decision analysis", Fuzzy Sets and Systems, vol. 90, pp. 199-206, 1997.

[18] J. Kacprzyk, M. Fedrizzi and H. Nurmi, "Group decision making and consensus under fuzzy preferences and fuzzy majority", Fuzzy Sets and Systems, vol. 49, pp. 21-31, 1992.

[19] C. Carlsson, D. Ehrenberg, P. Eklund, M. Fedrizzi, P. Gustafsson, P. Lindholm, G. Merkuryeva, T. Riissanen and A. G. S. Ventre, "Consensus in distributed soft environments", European Journal of Operational Research, vol. 61, pp. 165-185, 1992.

[20] L. Coch and J. R. P. French, "Overcoming resistance to change", Human Relations, vol. 1, pp. 512-532, 1948.

[21] J. R. P. French, "A formal theory of social power", Psychological Review, vol. 63, pp. 181-194, 1956.

[22] F. Harary, "On the measurement of structural balance", Behavioral Science, vol. 4, pp. 316-323, 1959.

[23] E. Herrera-Viedma, F. Herrera and F. Chiclana, "A consensus model for multiperson decision making with different preference structures", IEEE Transactions on Systems Man and Cybernetics Part A - Systems and Humans, vol. 32, 394-402, 2002.

[24] E. Herrera-Viedma, S. Alonso, F. Chiclana and F. Herrera, "A consensus model for group decision making with incomplete fuzzy preference relations", IEEE Transactions on Fuzzy Systems, vol. 15, 863-877, 2007.

[25] F. Mata, L. Martinez and E. Herrera-Viedma, "An adaptive consensus support model for group decision-making problems in a multigranular fuzzy linguistic context”, IEEE Transactions on Fuzzy Systems, vol. 17, 279-290, 2009

[26] S. Alonso, I. J. Pérez, F. J. Cabrerizo and E. Herrera-Viedma, "A linguistic consensus model for web 2.0 communities", Applied Soft Computing, vol. 13, 149-157, 2013.

[27] P. Victor, C. Cornelis, M. D. Cock and E. Herrera-Viedma, "Practical aggregation operators for gradual trust and distrust", Fuzzy Sets and Systems, vol. 184, 126-147, 2011.

[28] P. Victor, C. Cornelis, M. D. Cock and P. da Silva, "Gradual trust and distrust in recommender systems", Fuzzy Sets and Systems, vol. 160, 1367-1382, 2009.

[29] M. Tavana and D. T. Kennedy, "N-site: a distributed consensus building and negotiation support system", International Journal of Information Technology \& Decision Making, vol. 5, pp. 123-154, 2006.

[30] J. Kacprzyk, S. Zadrozny and Z. W. Ras, "How to support consensus reaching using action rules: a novel approach", International Journal of Uncertainty Fuzziness and Knowledge-Based Systems, vol. 18, pp. 451$470,2010$.

[31] F. Chiclana, E. Herrera-Viedma, F. Herrera and S. Alonso, "Some induced ordered weighted averaging operators and their use for solving group decision making problems based on fuzzy preference relations", European Journal of Operational Research, vol. 182, pp. 383-399, 2007.

[32] F. Herrera, E. Herrera-Viedma and J. L. Verdegay, "Choice processes for non-homogeneous group decision making in linguistic setting", Fuzzy Sets and Systems, vol. 94, pp. 287-308, 1998.

[33] F. Herrera, E. Herrera-Viedma and J. L. Verdegay, "A model of consensus in group decision making under linguistic assessments", Fuzzy Sets and Systems, vol. 78, pp. 73-87, 1996.

[34] F. Herrera, E. Herrera-Viedma and J. L. Verdegay, "A rational consensus model in group decision making using linguistic assessments", Fuzzy Sets and Systems, vol. 88, pp. 31-49, 1997.

[35] H. S. Lee, Optimal consensus of fuzzy opinions under group decision making environment, Fuzzy Sets and Systems, vol. 132, pp. 303-315, 2002.

[36] T. L. Saaty, "Time dependent decision-making; dynamic priorities in the AHP/ANP: generalizing from points to functions and from real to complex variables", Mathematical and Computer Modeling, vol. 46, pp. 860-891, 2007

[37] R. Cialdini, "The science of persuasion", Scientifc American, vol. 284, pp. 76-81, 2001. 